\title{
Potential benefits of intra-prostatic cancer-specific imaging to guide therapy and monitor outcome in patients treated with radiation-based treatments for localized
} prostate cancer

\author{
Ronald D. Ennis* \\ Director, Department of Radiation Oncology, St. Luke's-Roosevelt Hospital Center, NY, USA \\ Associate Director, Continuum Cancer Centers of New York, NY, USA \\ Associate Professor, Radiation Oncology, Albert Einstein College of Medicine, NY, USA
}

Keywords: Imaging, prostate cancer, adenocarcinoma of the prostate, differential treatment, focal treatment

\section{Benefits of intra-prostatic cancer-specific imaging for radiation therapy}

Prostate cancer is a multifocal disease in $76-87 \%$ of patients $[1,4,8]$. Typically, 3 separate foci of cancer are seen in a prostate which has been surgically removed, but there may be as many as twelve $[1,4$, 8]. Standard prostate biopsies cannot reliably detect these foci [7]. Ultrasound imaging, in expert hands, has a receiver operator characteristic curve of 0.6 in determining whether a region of the prostate contains cancer [3]. MRI is marginally better at detecting the separate intraprostatic foci and CT is completely ineffective for such imaging [7]. (MR spectropscopy shows great promise and is discussed in this issue of Cancer Biomarkers.) Therefore, in contrast to most

*Address for correspondence: Radiation Oncology, St. Luke's Roosevelt Hospital Center, 1000 Tenth Avenue, Lower Level, New York, NY 10019, USA. Tel.: +1 212523 7165; Fax: +1 212523 8189; E-mail: rennis@chpnet.org. other malignancies, all standard treatments for prostate cancer are applied to the entire prostate gland. This results in the exposure of the adjacent normal tissues to potential damage even if that tissue is not actually near any cancer. Radiation oncologists and urologists have devised methods to minimize damage to adjacent normal tissues such as peripheral loading of seeds in prostate brachytherapy, intensity modulated radiotherapy and nerve sparing prostatectomy. However, further sparing of normal tissues could be achieved if the treating physician could know with confidence that no (or little) cancer existed near a adjacent tissue based on a reliable imaging technique.

As an example, patients treated with any externalbeam technique receive doses of radiation that are uniformly distributed throughout the prostate. Consequently, the cancer-free regions receive the same dose as cancer-containing regions. The irrationality of this treatment method is obvious. With the ability to know where the cancer is, radiation oncologists could deliver higher doses to the tumor foci and less (or none) to 
the non-cancerous tissues. A similar concept applies to brachytherapy. Prostate brachytherapy delivers a less homogeneous dose than external-beam radiation does; however, the hot spots in brachytherapy are more or less randomly distributed throughout the prostate, with care taken to minimize the urethral dose. Again, with knowledge of the location of the cancer provided by improved imaging, a more rational approach to planning the seed locations could be undertaken.

Furthermore, extensive research has been undertaken in radiation oncology regarding the optimal dose for treating prostate cancer. For external-beam radiation, this research has found that an escalation of the dose improves the likelihood of being free of prostate cancer in the future. For brachytherapy, a specific prescribed dose is considered optimal for cancer control, and postimplant analysis is performed to assure the prescribed dose is achieved. However, all dose-response data are based on delivering the prescribed dose to the entire prostate gland, and are not based on the dose delivered to the tumor foci. Therefore, these data can serve only as imprecise guides. With the ability to image cancer foci reliably, a reevaluation of the dose response of prostate cancer, whether treated with external-beam techniques or brachytherapy, will be possible. This new analysis will be much more robust in its ability to predict successful treatment and will allow improved decision making by radiation oncologists.

Exploitation of improved intraprostatic imaging to move away from uniform treatment of the entire gland could be done in two ways. One method, termed differential treatment, would differentiate the treatment intensity, delivering more treatment to regions of larger cancer volumes, lesser treatment to areas of smaller cancer volumes, and lesser treatments still, to those areas where the imaging does not show cancer, but where malignant cells may exist in volumes below the level of detection of the imaging modality. The second method, termed partial (or focal) treatment, would only partially treat the prostate gland, treating only the regions identified as having cancer on the imaging and leaving the other parts of the gland completely untreated. Underlying this treatment approach is the assumption that small foci of cancer that are not detected with these new imaging modalities are not clinically relevant and will not progress even if left untreated.

To achieve any of these improvements, a highly reliable imaging method is needed. The ROC curve of this imaging should be substantially better than the ROC curves achieved with current clinically used ultrasound or MRI. In addition, determination of the smallest vol- ume of cancer that can be imaged with a particular technique is crucial to address the concerns regarding leaving any significant focus of cancer untreated. Until a technique can image on the cellular level, a risk of missing a small focus of cancer will remain. Knowledge of the minimal detectable cancer volume is crucial applying any new imaging technique properly in the differential treatment approach.

Radiotherapy of prostate cancer, whether using brachytherapy or external beam techniques, is exquisitely well suited for the differential treatment approach, allowing the radiation oncologist to exploit a new imaging technique even if it imperfectly detects prostate cancer foci because of the ability of these methods to deliver differential doses. A radiation dose distribution can be tailored to the volume of cancer in the different regions of the prostate. That is, a low dose can be given to regions where the scan detects no cancer, but a risk of a small volume of cancer remains. Moderate doses can be given to small regions of cancer detected by the new imaging technique and still higher doses can be delivered to regions of larger bulk of cancer. This ability to match treatment intensity to volume of cancer is unique to radiation and will allow the maximum exploitation of new imaging techniques in the differential treatment approach.

All other treatment modalities used in prostate cancer, such as surgery, cryotherapy and high intensity focused ultrasound are all-or-nothing therapies. These could be used for partial prostate treatment, but not for differential treatment. While focal treatments with methods other then radiation therapy would fully treat the large-volume foci, they would leave small, undetected volumes of prostate cancer untreated, and would not permit intermediate treatment to regions of lesser cancer volumes. Therefore, these techniques cannot be used for differential treatment and therefore cannot fully exploit the advances in imaging described in this special issue of Cancer Biomarkers until those imaging methods can reliably image prostatic tissue at the level of individual cells.

In addition to guiding more precise, tissue-sparing treatment, new imaging techniques may be helpful in monitoring patients after treatment. Currently, the standard for monitoring radiation-treated patients after therapy is palpation, termed a digital rectal exam (DRE), and a blood test termed a serum prostate-specific antigen (PSA) measurement. The DRE is very crude and rarely helpful. The literature regarding the utility of serum PSA monitoring post-treatment demonstrates an accuracy of no better than $\sim 70 \%$ [6]. The only defini- 
tive method available to assess the prostate itself is a prostate biopsy. This invasive test is a strong predictor of outcome, but because of sampling limitations is not a perfect one and is prone to a high false-negative rate [2,5]. A non-invasive means of monitoring success after any localized treatment that leaves the prostate in place is needed. This need will be particularly acute for patients undergoing treatment with the techniques that involve differential or partial treatment. Hopefully, the new imaging modalities discussed in this special issue of Cancer Biomarkers will improve our ability to assess the success of treatment in a reliable, non-invasive manner.

\section{References}

[1] R. Arora, M.O. Koch, J.N. Eble, T.M. Ulbright, L. Li and L. Cheng, Heterogeneity of Gleason Grade in Multifocal Adenocarcinoma of the Prostate, Cancer 100 (2004), 2362-2366.

[2] J.M. Crook, G.A. Perry, S. Robertson and B.A. Esche, Routine Prostate Biopsies Following Radiotherapy for Prostate Cancer: Results of 226 Patients, Urol 45 (1995), 624-632.

[3] E.J. Feleppa, R.D. Ennis, P.B. Schiff, C.S. Wuu, A. Kalisz, J. Ketterling, S. Urban, T. Liu, W.R. Fair, C.R. Porter and J.R. Gillespie, Ultrasonic Spectrum-analysis and Neural-network
Classification as a basis for Ultrasonic Imaging to Target Brachytherapy of Prostate Cancer, Brachytherapy 1 (2002), 4853.

[4] M. Noguchi, T.A. Stamey, J.E. McNeal and R. Nolley, Prognostic factors for Multifocal Prostate Cancer in Radical Prostatectomy Specimens: Lack of Significance of Secondary Cancers, J Urol 170 (2003), 459-463.

[5] A. Pollack, G.K. Zagars, J.A. Antolak, D.A. Kuban and I.I. Rosen, Prostate Biopsy Status and PSA nadir Level as Early Surrogates for Treatment Failure: Analysis of a Prostate Cencer Randomized Radiation Dose Escalation Trial, Int J Radiat Oncol Biol Phys 54 (2002), 677-685.

[6] H. Thames, D. Kuban, L. Levy, E.M. Horowitz, P. Kupelian, A. Martinez, J. Michalski, T. Pisansky, H. Snadler, W. Shipley, M. Zelefsky and A. Zeitman, Comparison of Alternative Biochemical Failure Definitions Based in Clinical Outcome in 4839 Prostate Cancer Patients Treated by External Beam Radiotherapy between 1986 and 1995, Int J Radiat Oncol Biol Phys 57 (2003), 929-943.

[7] A.E. Wefer, H. Hricak, D.B. Vigneron, F.V. Coakley, Y. Lu, J. Wefer, U. Mueller-Lisse, P.R. Carroll and J. Kurhanewicz, Sextant Localization of Prostate Cancer: Comparison of Sextant Biopsy, Magnetic Resonance Imaging and Magnetic Resonance Spectroscopy Imaging with Step Section Histology, $J$ Urol 164 (2000), 400-404.

[8] A.M. Wise, T.A. Stamey, J.E. McNeal and J.L. Clayton, Morphologic and Clinical Significance of Multifocal Prostate Cancers in Radical Prostatectomy Specimens, Urol 60 (2002), 264269. 